Epidemiol 1996;17(suppl):P25. Abstract 59.

6. Schentag JJ, Nix DE, Adelman MH. Mathematical examination of dual individualization principles. Relationships between AUC above MIC and area under the inhibitory curve (AUIC) for cefmenoxime, ciprofloxacin, and tobramycin. DICP 1991;25:1050-1057.

7. Forrest A, Nix DE, Ballow CH, Goss TF, Birmingham MC, Schentag JJ. Pharmacodynamics of intravenous ciprofloxacin in seriously ill patients. Antimicrob Agents Chemother 1993;
37:1073-1081

8. Holmberg SD, Solomon SL, Blake PA. Health and economic impacts of antimicrobial resistance. Rev Infect Dis 1987;9: 1065-1078.

9. Raad II, Hohn DC, Gilbrath J, et al. Prevention of central venous catheter-related infections by using maximal sterile barrier precautions during insertion. Infect Control Hosp Epidemiol 1994;15:231-238

\title{
Antimicrobial Resistance: Inpatients, Outpatients, and Role of the ICU
}

\section{Gina Pugliese, RN, MS; Martin S. Favero, PhD}

In 1994, the CDC's Hospital Infections Program, in cooperation with the Rollins School of Public Health at Emory University, initiated Project ICARE (Intensive Care Antimicrobial Resistance Epidemiology) to study the magnitude of the problem of antibioticresistant microorganisms among the various settings of acute-care hospitals, extended-care facilities, and ambulatory-care facilities. In addition, the role of the intensive-care unit (ICU) and its contribution to the overall problem of antibiotic resistance was a major element for research. The investigators recently presented data from phase I of the project. The objective was to assess the extent of antimicrobial resistance in hospitals, particularly in ICUs, compared with antimicrobial resistance found in the community, and to determine if pathogens isolated from inpatients are more resistant to antimicrobials than are pathogens from outpatients. Eight hospitals that participate in the ICU component of the National Nosocomial Infection Surveillance System were selected, and the study was conducted between April 1994 and March 1995. The percentage of resistant isolates from inpatients was higher than that from outpatients for the following antimicrobial/organism combinations: methicillin/coagulase-negative Staphylococcus (49\% vs 36\%); methicillin/Staphylococcus aureus (33\% vs 14.5\%); ceftazidime/Enterobacter cloacae (26\% vs $12 \%)$; imipenem/Pseudomonas aeruginosa (12\% vs $6.5 \%)$; ceftazidime/P aeruginosa $(7.8 \%$ vs $4 \%$ ); and vancomycin/Enterococcus species $(6.3 \%$ vs $1.4 \%)$. There was a significant stepwise decrease in the percentage of resistant organisms isolated from ICU patients, non-ICU inpatients, and outpatients, respectively.

The authors note that these data support the need for control of antimicrobial resistance to be focused in the ICU. Patients in the ICU are at the greatest risk of nosocomial infection, partly because of their serious underlying disease, but also because of exposure to life-saving invasive procedures, prolonged use of in-situ invasive devices, therapy with multiple antimicrobials, and extended hospital stays. In addition, antimicrobial resistance in pathogens is more likely to be encoun- tered in the ICU because of the selection effect of treatment with multiple antimicrobials for a single patient, which may result in amplification of antimicrobial resistance in organisms. Although the data found the hospital to be the focus of antimicrobial resistance, there were exceptions. An increasing number of ciprofloxacin-resistant Escherichia coli and imipenem-resistant $P$ aeruginosa among isolates from outpatients of one study hospital suggested some type of selection effect on this isolates. Further discussions with the infection control department revealed that most of these outpatient isolates came from the urinary tract of patients residing in nursing homes and long-term rehabilitation facilities. The authors recommend that more resources be allocated to control antimicrobial resistance, particularly in the ICU, including more surveillance activities, strict infection control, and improved antibiotic usage.

FROM: Archibald L, Phillips L, Monnet D, McGowan J, Tenover F, Gaynes R. Antimicrobial resistance in isolates from inpatients and outpatients in the United States: increasing importance of the intensive care unit. Clin Infect Dis 1997;24:211-215. 